\title{
Modeling Philippine Stock Exchange Composite Index Using Weighted Geometric Brownian Motion Forecasts
}

\author{
Willy Gayo ${ }^{1}$ and Guido David ${ }^{2}$ \\ ${ }^{1}$ College of Science, Polytechnic University of the Philippines, Manila, Philippines \\ ${ }^{2}$ Institute of Mathematics, University of the Philippines, Quezon City, Philippines
}

\begin{abstract}
Philippine Stock Exchange Composite Index (PSEi) is the main stock index of the Philippine Stock Exchange (PSE). PSEi is computed using a weighted mean of the top 30 publicly traded companies in the Philippines, called component stocks. It provides a single value by which the performance of the Philippine stock market is measured. Unfortunately, these weights, which may vary for every trading day, are not disclosed by the PSE. In this paper, we propose a model of forecasting the PSEi by estimating the weights based on historical data and forecasting each component stock using Monte Carlo simulation based on a Geometric Brownian Motion (GBM) assumption. The model performance is evaluated and its forecast compared is with the results using a direct GBM forecast of PSEi over different forecast periods. Results showed that the forecasts using WGBM will yield smaller error compared to direct GBM forecast of PSEi.
\end{abstract}

\section{Introduction}

The Philippine Stock Exchange Composite Index (PSEi), formerly known as Phisix, provides investors and other market participants a point of reference that can measure the performance of Philippine stock market. It is composed of 30 common stocks of listed companies, which are carefully selected by the Philippine Stock Exchange (PSE). To be selected, a company must rank among the top $25 \%$ in terms of median daily value in nine out of the twelve-month period in review, and have at least $12 \%$ free float level. The Philippine Stock Exchange (PSE) chooses the top 30 among qualified companies based on the full market capitalization. PSEi serves as an indicator in the price level changes of the entire Philippine stock market. It also provides a useful benchmark against which to measure an investor's portfolio. Because of these, there is a need to model and forecast the value of PSEi.

There have been different methods in modeling stock composite indices. Cointegration and Granger causality tests were used by Albu [1] in choosing what variables or indicators estimated the composite index of economic activity in Romania. Hussain and Li [2] used generalized extreme value (GEV), generalized logistic (GL) and generalized Pareto distributions to model the distribution of the extreme daily returns of the Shanghai Stock Exchange Composite Index from 1991 to 2013. They also used power-weighted method to estimate their model parameters. Leigh, Hightower and Modani [3] used a nonlinear neural network model that featured information on volume increase, interest rate change and previous price behavior in modeling the New York Stock Exchange (NYSE) Composite Index.

There were also other papers that studied PSEi. Sudirman and Darmayanti [4] used Box Jenkins methodology to estimate the best model for the stock exchange composite indices of Indonesia, Malaysia, Singapore, Philippines and Thailand. Dolores [5] applied Monte Carlo simulations based on a regime-switching lognormal model to project returns of PSEi. To estimate the parameters of the model, he used maximum likelihood estimation. He also compared the results obtained to the results using a simple lognormal model.

Stock prices and stock indices are stochastic in nature, which means they are not constant over time. Here, we model PSEi using Geometric Brownian Motion (GBM). Moreover, PSEi values are calculated based on a weighted mean of the component stocks. PSE is the governing agency that assigns the weight of each component stock. These weights are periodically subject to review and revision. Unfortunately, these weights are not publicly disclosed.

In this paper, we model and forecast PSEi using Monte Carlo simulations based on weighted GBM forecasts. The weights of the component stocks are estimated using constrained multilinear regression. We refer to this method as WGBM. The forecasts of WGBM are then compared with those obtained by modeling PSEi as GBM, referred to as GBM.

\section{Methods}


Let $t=0,1,2, \ldots, T$ where $T$ is the end horizon, $W_{t}$ be a Brownian motion and $\mathcal{F}_{t}, t \geq 0$, be an associated filtration. Define a geometric Brownian process by the stochastic differential equation

$$
d S_{t}=\alpha S_{t} d t+\sigma S_{t} d W_{t} .
$$

By Itô's formula, assuming constant $\alpha, \sigma$, (1) can be solved as

$$
S_{t}=S_{0} \exp \left(\left(\alpha-\frac{1}{2} \sigma^{2}\right) t+\sigma W_{t}\right) .
$$

The parameters $\alpha$ and $\sigma$ are the drift rate and volatility of the GBM, respectively.

Let $Y_{t}=\log \left(S_{t} / S_{t-1}\right)$ for $t=1,2, \ldots, T$. Then the volatility $\sigma$ is

$$
\sigma=\operatorname{std}\left(Y_{t}\right) \times \sqrt{N}
$$

where $\operatorname{std}\left(Y_{t}\right)$ is the standard deviation of $Y_{t}$ for $t=$ $1,2, \ldots, T$ and $N$ is the number of trading days in a year. The drift of $S_{t}$ is calculated using linear regression on the $\log$ price process, i.e. we seek the parameters $a$ and $b$ such that minimizes

$$
\varepsilon=\sum_{t=1}^{T}\left(\log S_{t}-b-a t\right)^{2} .
$$

The drift rate $\alpha$ is then obtained by

$$
\alpha=N a+\sigma^{2} / 2 \text {. }
$$

Let $S_{t}$ be the actual value, $\hat{S}_{t}$ be the forecast value and $e_{t}=S_{t}-\hat{S}_{t}$ be the forecast error or residual for each time $t=1,2, \ldots, T$. To evaluate the performance of the model, we use the root mean square error (RMSE) and mean absolute percentage error (MAPE). RMSE measures the square root of the average squared deviation of the forecasted and actual values, given by:

$$
R M S E=\sqrt{\frac{1}{T} \sum_{t=1}^{T} e_{t}^{2}} .
$$

MAPE measures the percentage of average absolute deviation of the forecasted and actual values, given by:

$$
M A P E=\frac{1}{T} \sum_{t=1}^{T} \frac{\left|e_{t}\right|}{S_{t}} .
$$

Let $i=1,2, \ldots, n$ be the index of the component stock. Given $P_{t}$ the PSEi value at time $t, S_{t}^{i}$ and $V_{t}^{i}$ the closing price and outstanding volume of stock $i$ at time $t$, respectively, and $w^{i}$ the weight of each stock $i$, then the weights of the component stocks are calculated using constrained multilinear regression, i.e. we solve

$$
\mathbf{P}=\mathbf{A w}
$$

for $\mathbf{w}$,subject to $w^{i} \geq 0$ for $i=1,2, \ldots, n$. Here the entries of the design matrix $\mathbf{A}$ are given by $A_{t}^{i}=S_{t}^{i} \cdot V_{t}^{i}$ for each $t$ and $i$.

\section{Results}

We used historical data of the closing prices of the component stocks and PSEi for every trading day from 2012 to 2014. The first method (WGBM) modeled PSEi using100,000 Monte Carlo simulations of the 30 component stocks, each assumed to follow GBM process, repeated 30 times. In the second method (GBM), PSEi was modeled using Monte Carlo simulations of the PSEi index, assumed to follow GBM process, repeated 30 times. The Monte Carlo simulations were implemented using Matlab ${ }^{\circledR}$. We varied the range of historical data used and the forecast period to evaluate model performance.
Each component stock of PSEi was modeled and forecasted using WGBM where the parameters $\alpha$ and $\sigma$ were estimated using (5) and (3), respectively. The forecasted closing price of component stock $i$ for $i=1,2, \ldots, n$ was denoted by $\hat{S}_{t}^{i}$ for each time $t$. We then used (8)to determine the weight of each component stock. The forecasted value of PSEi, denoted by $\hat{P}_{t}$, was computed by

$$
\hat{P}_{t}=\sum_{i=1}^{n}\left(\hat{S}_{t}^{i} \cdot V_{t}^{i}\right) w^{i}
$$

For the first forecast period, we used PSEi data from April 2012 to December 2013 to model and forecast PSEi for January to March 2014. There were 411 trading days during this time frame. Table 1 shows the computed weight for each component stock where each weight was scaled by $10^{-8}$.

Table 1. Weights of the 30 component stocks $\left(\times 10^{-8}\right)$ for the period from April 2012 to December 2013.

\begin{tabular}{|c|c|c|c|c|c|}
\hline$i$ & Symbol & $w^{i}$ & $i$ & Symbol & $w_{i}$ \\
\hline 1 & AC & 0.1126 & 16 & JFC & 0.1569 \\
\hline 2 & AEV & 0.0507 & 17 & JGS & 0.0000 \\
\hline 3 & AGI & 0.0805 & 18 & LTG & 0.0129 \\
\hline 4 & ALI & 0.2328 & 19 & MBT & 0.1991 \\
\hline 5 & AP & 0.2159 & 20 & MEG & 0.0000 \\
\hline 6 & BDO & 0.1531 & 21 & MER & 0.0360 \\
\hline 7 & BLOOM & 0.0924 & 22 & MPI & 0.2224 \\
\hline 8 & BPI & 0.0519 & 23 & PCOR & 0.0005 \\
\hline 9 & DMC & 0.0961 & 24 & RLC & 0.2327 \\
\hline 10 & EDC & 0.2128 & 25 & SCC & 0.0000 \\
\hline 11 & EMP & 0.0000 & 26 & SM & 0.0075 \\
\hline 12 & FGEN & 0.2174 & 27 & SMC & 0.1482 \\
\hline 13 & GLO & 0.1304 & 28 & SMPH & 0.0000 \\
\hline 14 & GTCAP & 0.2082 & 29 & TEL & 0.0000 \\
\hline 15 & ICT & 0.3857 & 30 & URC & 0.1002 \\
\hline
\end{tabular}

The component stocks were individually modeled using the GBM process given in (2). Table 2 shows the drift rate $\left(\alpha^{i}\right)$ and volatility $\left(\sigma^{i}\right)$ of component stock $i$, for $i=1,2, \ldots, 30$.We then used these parameters to forecast each component stock for the period from January to March 2014.

Table 2. Drift rate and volatility of the component stocks.

\begin{tabular}{|c|c|c|c|c|c|}
\hline$i$ & $\alpha^{i}$ & $\sigma^{i}$ & $i$ & $\alpha^{i}$ & $\sigma^{i}$ \\
\hline 1 & 0.2474 & 0.3112 & 16 & 0.3870 & 0.3693 \\
\hline 2 & 0.0174 & 0.3345 & 17 & 0.1758 & 0.3119 \\
\hline 3 & 0.6014 & 0.3571 & 18 & 0.9135 & 0.5612 \\
\hline 4 & 0.2313 & 0.3569 & 19 & -0.0063 & 0.3165 \\
\hline 5 & -0.0116 & 0.2549 & 20 & 0.3877 & 0.3621 \\
\hline 6 & 0.1782 & 0.3267 & 21 & 0.1270 & 0.3066 \\
\hline 7 & 0.0147 & 0.4736 & 22 & 0.1361 & 0.3247 \\
\hline 8 & 0.2031 & 0.2816 & 23 & 0.2346 & 0.2492 \\
\hline 9 & -0.0941 & 0.3001 & 24 & 0.1635 & 0.3318 \\
\hline 10 & -0.0781 & 0.3376 & 25 & 0.1380 & 0.2907 \\
\hline 11 & 1.0194 & 0.8852 & 26 & 0.1112 & 0.3655 \\
\hline 12 & -0.0668 & 0.2934 & 27 & -0.2991 & 0.2952 \\
\hline 13 & 0.3180 & 0.3191 & 28 & 0.1570 & 0.3906 \\
\hline 14 & 0.3684 & 0.3071 & 29 & 0.0821 & 0.2147 \\
\hline 15 & 0.2779 & 0.3276 & 30 & 0.5471 & 0.3393 \\
\hline
\end{tabular}

Note that Emperador Inc. (EMP) had the highest drift rate and highest volatility, while San Miguel Corporation 
(SMC) had the lowest drift rate and was among six stocks that had negative drift rates, indicating a general downward trend over the period of study. Philippine Long Distance Telephone Company (TEL) had the lowest volatility. Using the estimated drift rate and volatility, the forecasted values of each component stock were multiplied by the corresponding outstanding volume (i.e. number of outstanding shares) and the weight computed using constrained multilinear regression in Table 1 to determine the predicted value of PSEi. Averaging over 100,000 runs and repeated 30 times, the forecasted values of PSEi using weighted Geometric Brownian Motion (WGBM) are shown in Figure 1. The performance of the forecasts was evaluated using RMSE and MAPE given by (6) and (7). The computed mean RMSE and MAPE are 132.1974 and $1.6717 \%$, respectively.

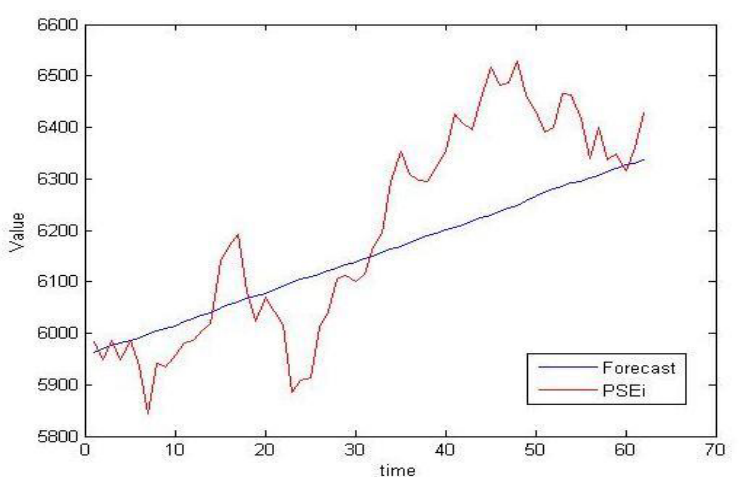

Figure 1.Graph of PSEi with WGBM forecasts from January to March 2014.

We extended the period of study to 2.25 years, 2.5 years, 2.75 years and 3 years and used WGBM to model the value of PSEi by recalculating the weight of each component stock using constrained multilinear regression, subject to nonnegative parameters. Then, we forecasted the values of PSEi for the succeeding quarter following the period of study. We conducted 100,000 runs each simulation, repeated 30 times and recorded the RMSE for each simulation. Afterwards, we computed the mean and standard deviation of the errors compared with the actual values of PSEi in the forecast period.

Table 3 shows the weight of each component stock obtained over the study period from April 2012 to March 2014. Figure 2 shows the forecast for April to June 2014 with mean RMSE and MAPE of 203.6851 and $2.5987 \%$, respectively.

Table 3. Weights of the 30 component stocks $\left(\times 10^{-8}\right)$ for the period from April 2012 to March 2014.

\begin{tabular}{|c|c|c|c|c|c|}
\hline$i$ & Symbol & $w^{i}$ & $i$ & Symbol & $w_{i}$ \\
\hline 1 & AC & 0.1189 & 16 & JFC & 0.2099 \\
\hline 2 & AEV & 0.1918 & 17 & JGS & 0.0301 \\
\hline 3 & AGI & 0.0016 & 18 & LTG & 0.0001 \\
\hline 4 & ALI & 0.1721 & 19 & MBT & 0.3115 \\
\hline 5 & AP & 0.0000 & 20 & MEG & 0.0001 \\
\hline 6 & BDO & 0.0000 & 21 & MER & 0.0343 \\
\hline 7 & BLOOM & 0.1015 & 22 & MPI & 0.2600 \\
\hline 8 & BPI & 0.1703 & 23 & PCOR & 0.0005 \\
\hline 9 & DMC & 0.0900 & 24 & RLC & 0.0005 \\
\hline
\end{tabular}

\begin{tabular}{|c|c|c|c|c|c|}
\hline 10 & EDC & 0.0798 & 25 & SCC & 0.0002 \\
\hline 11 & EMP & 0.0000 & 26 & SM & 0.0148 \\
\hline 12 & FGEN & 0.2704 & 27 & SMC & 0.1570 \\
\hline 13 & GLO & 0.1962 & 28 & SMPH & 0.0001 \\
\hline 14 & GTCAP & 0.8554 & 29 & TEL & 0.0000 \\
\hline 15 & ICT & 0.3858 & 30 & URC & 0.0005 \\
\hline
\end{tabular}

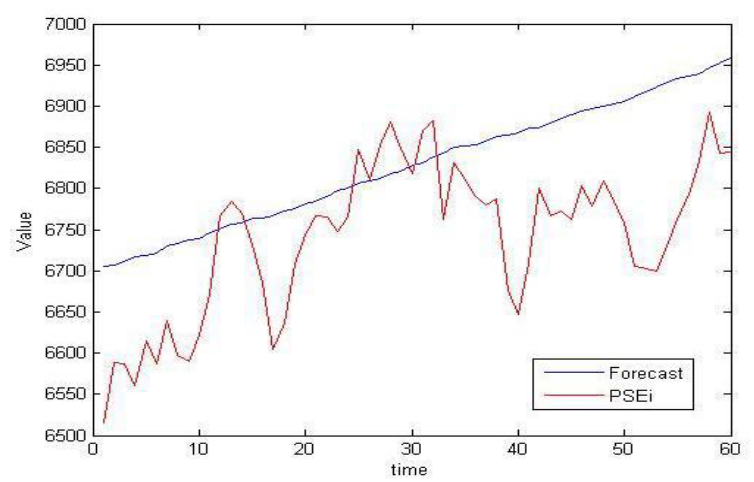

Figure 2. Graph of PSEi with WGBM forecasts from April to June 2014.

For the study period from April 2012 to June 2014, Table 4 shows the weight of each component stock and Figure 3 shows the forecast for July to September 2014. The mean RMSE and MAPE of the forecast are 191.0458 and $2.5645 \%$, respectively.

Table 4. Weights of the 30 component stocks $\left(\times 10^{-8}\right)$ for the period from April 2012 to June 2014.

\begin{tabular}{|c|c|c|c|c|c|}
\hline$i$ & Symbol & $w^{i}$ & $i$ & Symbol & $w_{i}$ \\
\hline 1 & AC & 0.0958 & 16 & JFC & 0.2473 \\
\hline 2 & AEV & 0.2172 & 17 & JGS & 0.0141 \\
\hline 3 & AGI & 0.0000 & 18 & LTG & 0.0000 \\
\hline 4 & ALI & 0.1532 & 19 & MBT & 0.3626 \\
\hline 5 & AP & 0.0000 & 20 & MEG & 0.0000 \\
\hline 6 & BDO & 0.0000 & 21 & MER & 0.0369 \\
\hline 7 & BLOOM & 0.0765 & 22 & MPI & 0.2317 \\
\hline 8 & BPI & 0.1986 & 23 & PCOR & 0.0000 \\
\hline 9 & DMC & 0.1138 & 24 & RLC & 0.0007 \\
\hline 10 & EDC & 0.0476 & 25 & SCC & 0.0000 \\
\hline 11 & EMP & 0.0000 & 26 & SM & 0.0214 \\
\hline 12 & FGEN & 0.3488 & 27 & SMC & 0.1131 \\
\hline 13 & GLO & 0.1943 & 28 & SMPH & 0.0000 \\
\hline 14 & GTCAP & 0.7567 & 29 & TEL & 0.0000 \\
\hline 15 & ICT & 0.3870 & 30 & URC & 0.0000 \\
\hline
\end{tabular}

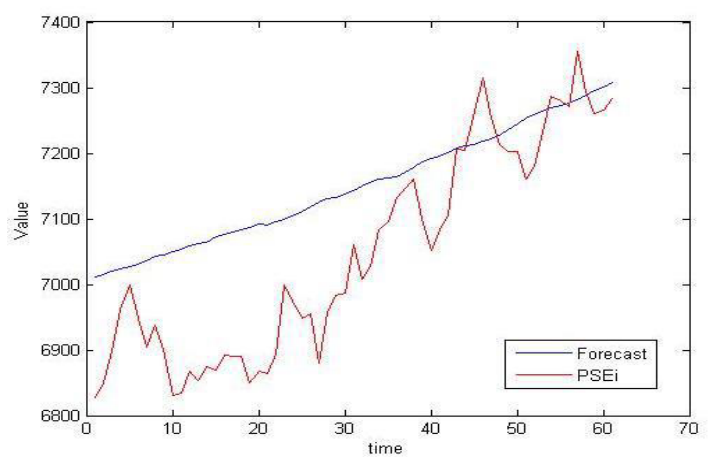

Figure 3. Graph of PSEi with WGBM forecasts from July to September 2014.

For the study period from April 2012 to September 2014, Table 5 shows the weight of each component stock 
and Figure 4 shows the forecast for October to December 2014. The mean RMSE and MAPE of the forecast are 97.4783 and $1.1812 \%$, respectively.

Table 5. Weights of the 30 component stocks $\left(\times 10^{-8}\right)$ for the period from April 2012 to September 2014.

\begin{tabular}{|c|c|c|c|c|c|}
\hline$i$ & Symbol & $w^{i}$ & $i$ & Symbol & $w_{i}$ \\
\hline 1 & AC & 0.0262 & 16 & JFC & 0.3167 \\
\hline 2 & AEV & 0.2769 & 17 & JGS & 0.0021 \\
\hline 3 & AGI & 0.0000 & 18 & LTG & 0.0001 \\
\hline 4 & ALI & 0.2003 & 19 & MBT & 0.1230 \\
\hline 5 & AP & 0.0000 & 20 & MEG & 0.0000 \\
\hline 6 & BDO & 0.0000 & 21 & MER & 0.0950 \\
\hline 7 & BLOOM & 0.0588 & 22 & MPI & 0.4486 \\
\hline 8 & BPI & 0.1711 & 23 & PCOR & 0.0000 \\
\hline 9 & DMC & 0.0067 & 24 & RLC & 0.0002 \\
\hline 10 & EDC & 0.2616 & 25 & SCC & 0.0000 \\
\hline 11 & EMP & 0.0000 & 26 & SM & 0.0451 \\
\hline 12 & FGEN & 0.1944 & 27 & SMC & 0.1519 \\
\hline 13 & GLO & 0.1564 & 28 & SMPH & 0.0000 \\
\hline 14 & GTCAP & 0.7407 & 29 & TEL & 0.0000 \\
\hline 15 & ICT & 0.2849 & 30 & URC & 0.0001 \\
\hline
\end{tabular}

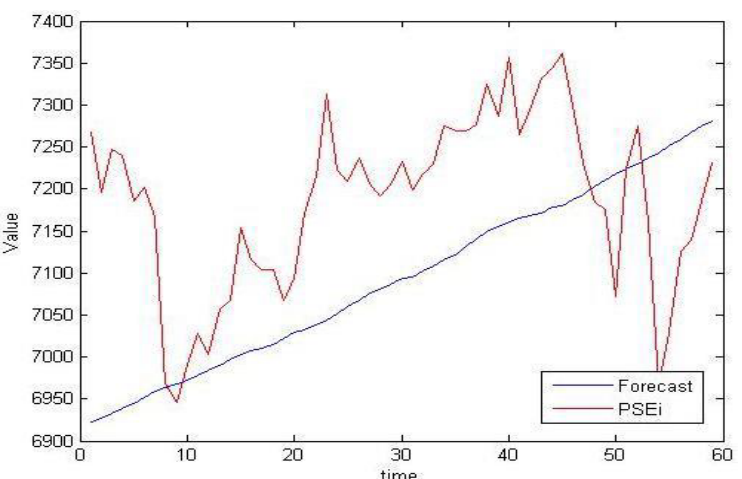

Figure 4. Graph of PSEi with WGBM forecasts from October to December 2014.

Lastly for the study period from April 2012 to December 2014, Table 6 shows the weight of each component stock and figure 5 shows the forecast for July to September 2014. The mean RMSE and MAPE of the forecast are 273.5275 and $3.3441 \%$, respectively.

Table 6. Weights of the 30 component stocks $\left(\times 10^{-8}\right)$ for the period from April 2012 to December 2014.

\begin{tabular}{|r|c|c|c|c|c|}
\hline$i$ & Symbol & $w^{i}$ & $i$ & Symbol & $w_{i}$ \\
\hline 1 & AC & 0.0628 & 16 & JFC & 0.2698 \\
\hline 2 & AEV & 0.2938 & 17 & JGS & 0.0000 \\
\hline 3 & AGI & 0.0000 & 18 & LTG & 0.0000 \\
\hline 4 & ALI & 0.2036 & 19 & MBT & 0.1393 \\
\hline 5 & AP & 0.0000 & 20 & MEG & 0.0000 \\
\hline 6 & BDO & 0.0000 & 21 & MER & 0.0881 \\
\hline 7 & BLOOM & 0.0424 & 22 & MPI & 0.4690 \\
\hline 8 & BPI & 0.2165 & 23 & PCOR & 0.0000 \\
\hline 9 & DMC & 0.0000 & 24 & RLC & 0.0000 \\
\hline 10 & EDC & 0.1213 & 25 & SCC & 0.0000 \\
\hline 11 & EMP & 0.0000 & 26 & SM & 0.0370 \\
\hline 12 & FGEN & 0.1977 & 27 & SMC & 0.1529 \\
\hline 13 & GLO & 0.1726 & 28 & SMPH & 0.0000 \\
\hline 14 & GTCAP & 0.4910 & 29 & TEL & 0.0000 \\
\hline 15 & ICT & 0.3232 & 30 & URC & 0.0000 \\
\hline
\end{tabular}

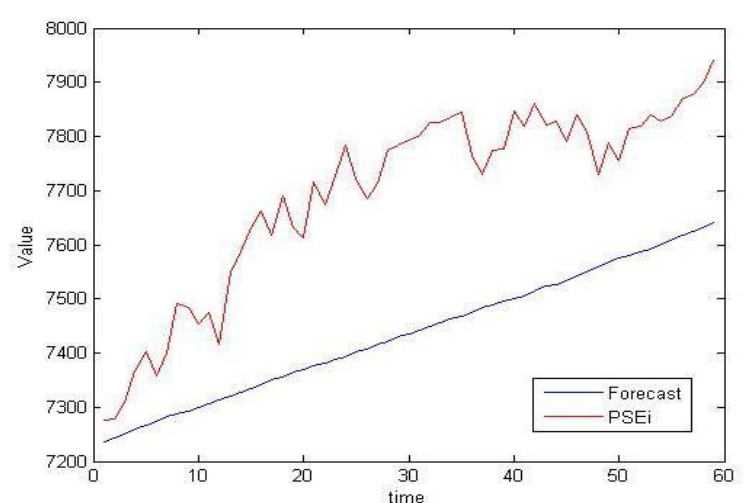

Figure 5. Graph of PSEi with WGBM forecasts from January to March 2015.

As comparison for WGBM, we then modeled PSEi directly using Monte Carlo simulations based on GBM. The drift and volatility of PSEi were computed similarly using (5) and (3). Table 7 shows the drift rate and volatility of the value of PSEi for the different periods of study. Figures 6 to 10 show the forecast for different periods of the study.

Table 7. Drift rate and volatility of PSEi modeled using GBM.

\begin{tabular}{|c|c|c|}
\hline Forecast Period & Drift Rate & Volatility \\
\hline 2 years & 0.1693 & 0.1972 \\
\hline 2.25 years & 0.1254 & 0.1902 \\
\hline 2.5 years & 0.1200 & 0.1828 \\
\hline 2.75 years & 0.1206 & 0.1757 \\
\hline 3 years & 0.1189 & 0.1727 \\
\hline
\end{tabular}

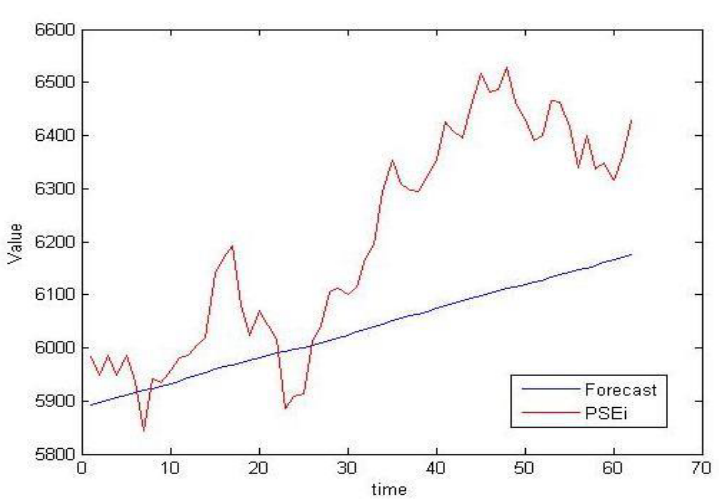

Figure 6. Graph of PSEi and forecast for January to March 2014 using GBM.

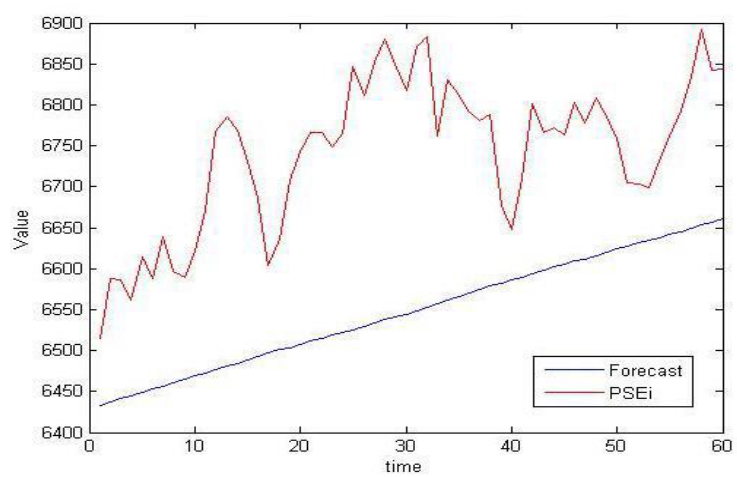

Figure 7. Graph of PSEi and forecast for April to June 2014 using GBM. 


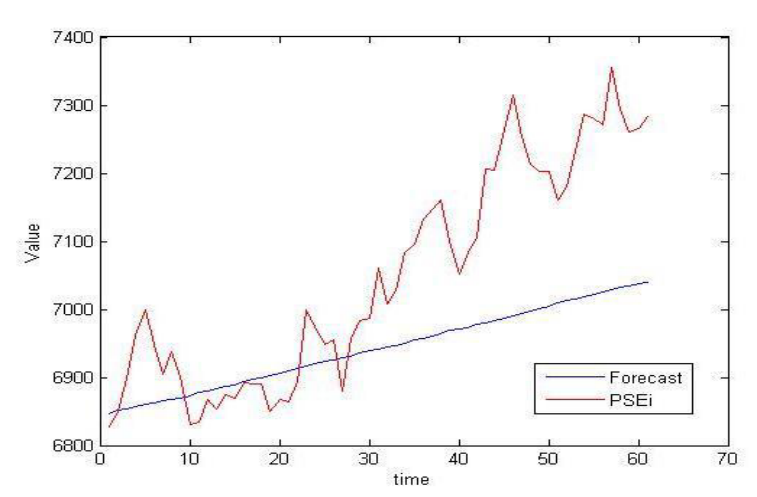

Figure 8. Graph of PSEi and forecast for July to September 2014 using GBM.

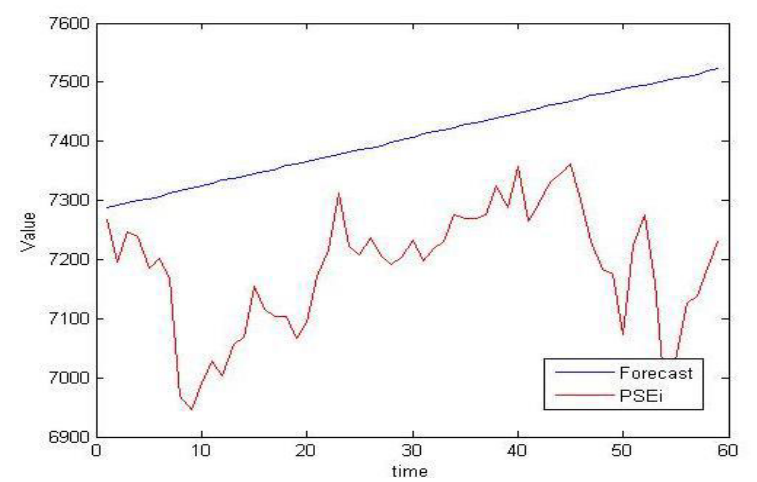

Figure 9. Graph of PSEi and forecast for October to December 2014 using GBM.

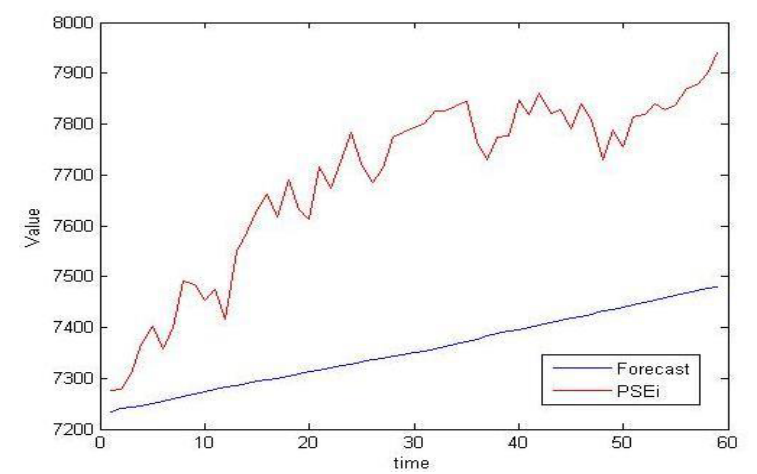

Figure 10. Graph of PSEi and forecast for January to March 2015 using GBM.

Table 8 shows the mean and standard deviation RMSE of the model performance over different forecast periods. WGBM has smaller RMSE compared to GBM for all forecast periods. Based on the results of this study, WGBM obtained better forecasts compared to directly using GBM on PSEi, with difference in errors as high as $100 \%$.
Table 8. Evaluation for different forecast periods using GBM.

\begin{tabular}{|c|c|c|}
\hline Forecast Period & RMSE & MAPE \\
\hline 2 years & 211.3857 & $2.7490 \%$ \\
\hline 2.25 years & 207.2595 & $2.8616 \%$ \\
\hline 2.5 years & 147.9104 & $2.6473 \%$ \\
\hline 2.75 years & 247.8183 & $3.1248 \%$ \\
\hline 3 years & 356.4535 & $4.3538 \%$ \\
\hline
\end{tabular}

\section{Conclusion}

In this paper, the Philippine Stock Exchange index was modeled using Monte Carlo simulations based on weighted Geometric Brownian Motion forecasts and a direct application of Geometric Brownian Motion. Simulations were done for 100,000 iterations and repeated 30 times. The performance of each model was evaluated using root mean square error. Numerical results showed that the first method (WGBM) yielded a significantly smaller RMSE compared to direct GBM. Hence, WGBM was a better method in modeling PSEi, in this study. WGBM method should be applicable to any stock index data, and even other stochastic data such as interest rates, exchange rates, mutual funds and others. It may also be used in weather data such as precipitation, rainfall and others. This study may be extended further using other stochastic models for forecasting. The estimation of the weights of the component stocks may also be improved.

\section{References}

1. L.L. Albu, RJEF 9 Issue 4,111-124 (2008)

2. S.F. Hussain, S. Li, Int. Fin. Markets, Inst. and Money 34, 263-276 (2015)

3. W. Leigh, R. Hightower, N. Modani, Expert Systems with Applications 28, 1-8 (2005)

4. M.S.N. Sudirman, P.A. Darmayanti, Conference Paper in Forum Manajemen Indonesia ke-5 (2014)

5. P.A. Dolores, Technical Paper (University of the Philippines)

6. S.E. Shreve, Stochastic Calculus for Finance II: Continuous-Time Model, Springer Finance, United States of America, (2004)

7. S.M. Ross, An Elementary Introduction to Mathematical Finance 3rd Ed., Cambridge University Press, (2011) 